\title{
Simulation of an Ondes Martenot Circuit
}

\author{
Judy Najnudel ${ }^{\dagger}$, Thomas Hélie*, David Roze*, Henri Boutin ${ }^{\dagger}$ \\ *S3AM Team, STMS Laboratory (UMR 9912), CNRS-IRCAM-SU \\ ${ }^{\dagger}$ S3AM Team, STMS Laboratory (UMR 9912), SU-IRCAM-CNRS
}

\begin{abstract}
The ondes Martenot is a classic electronic musical instrument based on heterodyning processing. This paper proposes a power-balanced simulation of its circuit, in order to synthesize the sound it produces. To this end, the proposed approach consists in formulating the circuit as a Port-Hamiltonian System, for which power-balanced numerical methods are available. Observations on numerical experiments based upon this formulation allow simplifications of the circuit in order to achieve real-time computation in home-studio conditions.
\end{abstract}

Index Terms-Electronic music instrument, non-linear, powerbalanced, simulation

\section{INTRODUCTION}

A $\mathrm{S}$ the audio industry is evolving from analog to digital, the preservation of analog machines and instrumentsand with them, that of the craft of generations of engineersis proving critical [1], [2]. The concern has been especially raised about the ondes Martenot; invented in 1928 by Maurice Martenot, this pioneer electronic musical instrument [3] is no longer produced in its original form and some of its components are now obsolete, such as specific triode vacuum tubes. A solution consists of modeling its circuit and building a real-time virtual instrument, so that the community of composers, musicians and musicologists may have access to virtual facsimiles.

Several techniques are available to build virtual analog instruments (for a review, see [4]-[6]). They include Modified Nodal Analysis [7], [8], the Nodal DK method [9] (for audio applications, see e.g. [10], [11]), Wave Digital Filters [12] (see e.g. [13]-[19]). Real-time simulations of circuits with vacuum tubes have been derived from such methods [20], [21] or from time-continuous state-space representations combined with numerical schemes [22]-[25]. The approach used in this paper is based on a state-space representation, which satisfies the power balance of the physical system structured into conservative, dissipative and external parts, known as PortHamiltonian Systems (referred to as PHS) [26], [27]. This formulation can be combined with numerical methods that preserve the power balance structure (and passivity) in the discrete-time domain for both linear and non-linear systems. This has proved to be relevant for simulations of audio (electronic or multi-physical) systems [28]-[31]. In a previous paper [32], the first stage of the ondes Martenot circuit (a variable oscillator) has been modeled and simulated using this method, with a special focus on electro-mechanical power balance. This paper addresses the full modeling and simulation of the circuit of the ondes Martenot No. 169, which is composed of 5 coupled stages. Moreover, analyzing numerical experiments allows some model simplifications, which in turn grant access to real-time.
This paper is organized as follows. Section II describes the history and the composition of the ondes Martenot as well as the way the instrument is played. In section III, the PHS formalism is briefly recalled and illustrated with a simple example. In section IV, each isolated stage is first separately modeled as a PHS and the coupling of the stages is then addressed. In section $\mathrm{V}$, the physical parameters for simulation are examined and set. Section VI is devoted to off-line powerbalanced simulations of the complete circuit. Finally, in section VII, simplifications are proposed based on the analysis of numerical experiments. They lead to a reduced order PHS from which a real-time audio plug-in is built for the sound synthesis.

\section{History, ORGANOLOGY, CIRCUIT AND PRINCIPLE}

\section{A. History}

The advent of electrical engineering marked a disruption in many industrial domains, amongst them musical instrument making. Providing both new timbres and new playing modalities, it gave the 20th century instrument corpus its singularity. Nonetheless, instrument making is not immune to technological obsolescence, and only few of these innovative instruments are still played today. This is the case of the ondes Martenot; only 264 ondes were built, but around 1500 pieces were composed for it [33]. As they were handmade and subjected to successive enhancements, each ondes is unique and identified by a reference number. The considerable task of cataloguing and evaluating the condition of the remaining instruments has been achieved by the Musée de la Musique in Paris [34]. It appears that for some of the ondes, making them playable again would necessitate irreversible interventions. This poses a big deontological dilemma from the preservation point of view and argues in favor of building facsimiles.

\section{B. Organology}

This paper focuses on the ondes No. 169, manufactured in 1937, and kept in the Musée de la Musique in Paris. This specimen has a great heritage value, particularly because of its electronic circuit with vacuum triodes. Also, it is kept in playing conditions and is fully documented [35]. It is based on heterodyne processing, like the Theremin [36] and the Trautonium [37]. It generates a harmonic signal which feeds specific loudspeakers (called diffuseurs, Fig. 1a). The player controls the pitch with a sliding ribbon (continuous pitch change) or-in later versions of the instrument-with a mobile keyboard (discrete pitch change and vibratos, Fig. 1b). The loudness is controlled with an intensity key, by pressing a small bag containing a mixture of conductive and insulating 


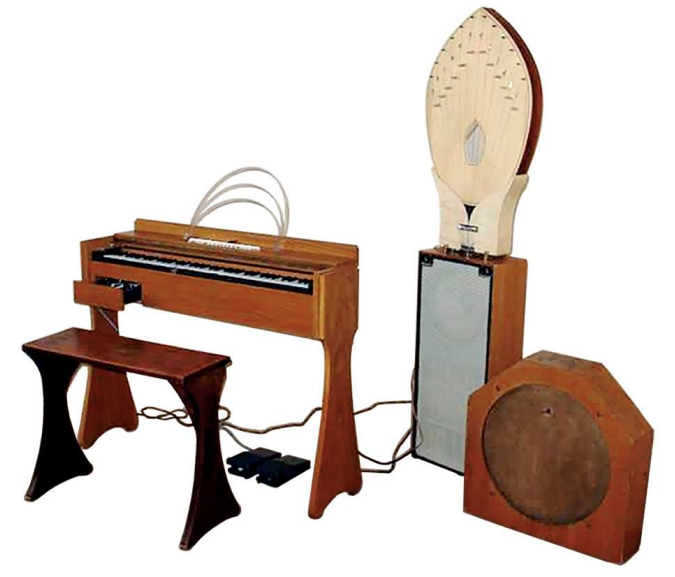

(a) (b)

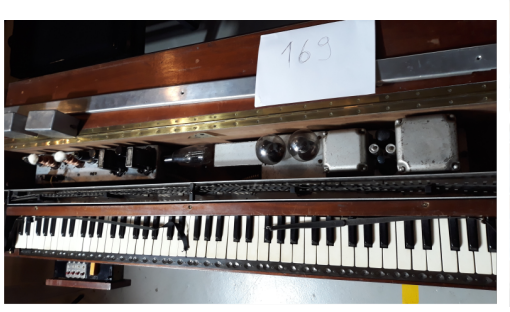

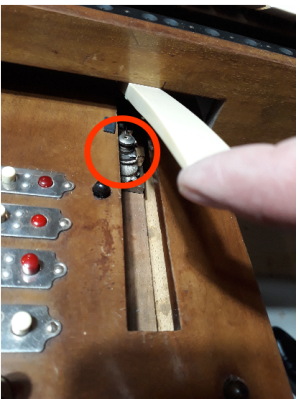

(c)
Fig. 1: Different parts of the ondes Martenot : diffuseurs (Fig. 1a on the right, from [33]), circuit, ribbon, keyboard (Fig. 1b), intensity key with the powder bag underneath circled in red (Fig. 1c).

powders. This key operates as a rheostat [38] (Fig. 1c). Thanks to this intensity key, the instrument's expressiveness has been praised since its creation. However, the lack of robustness of its circuit (subject to drifting, particularly in older models) is dreaded by musicians (called ondists) who are accustomed to perform repairs on the fly before concerts.

\section{Circuit and operation}

The technique of heterodyning was developed originally for wireless telegraphy [39], in order to shift high frequency signals into the audio domain. In the ondes Martenot specifically, two oscillators generate quasi-sinusoidal voltages at high frequency; one is set to $80 \mathrm{kHz}$ and the other, variable below $80 \mathrm{kHz}$, is controlled by the player. Their sum is an amplitude-modulated signal, whose envelope is detected with a triode vacuum tube in series with an $\mathrm{RC}$ circuit. The envelope fundamental frequency, equal to the difference between the oscillators' frequencies, lies in the audible frequency range. The resulting voltage is amplified through two successive triode vacuum tubes, which increase the harmonic distortion present in the signal, due to their non-linear characteristics.

Finally, the diffuseur, selected by the player, converts the electrical waveform into sound and in turn modifies its spectral content.

The complete circuit is structured into five stages devoted to specific functionalities (see Fig. 2a), namely : (1) fixedfrequency oscillator, (2) variable-frequency oscillator, (3) demodulator, (4) preamplifier, and (5) power amplifier. These stages are connected through transformers that introduce coupling: this will be naturally taken into account in the global PHS model (addressed in section IV-C). However, in first step, these stages are presented separately, ignoring coupling (no load on the secondary winding, 2b-2d). Qualitative specificities of these stages are stated below.

1) Oscillators: In both oscillators, a voltage noise $V_{\text {start }}$ at the triode grid is amplified by the triode vacuum tube and filtered by a LC circuit, before feeding the grid through a transformer of ratio $P$. Each transformer has two windings on the secondary; the second secondaries (for oscillators 1 and 2) are connected in series to form the demodulator voltage input.

2) Demodulator: The voltage input, equal to the sum of the oscillators' outputs, can be assimilated to an amplitudemodulated sinewave: $\cos (\Phi)+\cos \left(\Phi-\phi_{m}\right)=2 \cos (\Phi-$ $\left.\phi_{m} / 2\right) \cos \left(\phi_{m} / 2\right)$, where $F=\dot{\Phi} / 2 \pi=80 \mathrm{kHz}$ is the fixed oscillator frequency, $f_{m}=\dot{\phi}_{m} / 2 \pi$ is the target frequency, and $F-f_{m}$ is the variable oscillator frequency. The demodulator is composed of a triode vacuum tube in series with an $\mathrm{RC}$ circuit ( $R_{4}$ and $C_{21}$ on Fig. 2c). As the triode grid polarization is close to zero, the qualitative behavior of the triode between the grid and the cathode is that of a diode: grid current only appears for positive grid voltages (Fig. 3). The triode vacuum tube is loaded with capacitors in parallel with a transformer. This load forms a resonant bandpass RLC filter (Fig. 2c): the resistance $R_{\mathrm{p}}$ models the transformer losses, $L_{\mathrm{p}}$ is the primary inductance, $C_{\mathrm{dem}}$ is the equivalent capacitor of $C_{9}$ and $C_{10}$ in parallel. Its resonance frequency lies in the audible range. The stage amplification in a common cathode pattern increases with the triode load. As a consequence, the oscillators' frequencies and their intermodulation products are filtered out before amplification.

3) Preamplifier and power amplifier: Both stages rely on the triode vacuum tube amplification properties in a common cathode pattern [40].

\section{POWER-BALANCED MODELING}

\section{A. Framework}

Detailed presentations of PHS are available in [27]. Here we rely on a differential-algebraic formulation adapted to multiphysical systems [30], which allows the representation of a dynamical system as a network of

1) components storing energy $E$ as a regular definite nonnegative function of their state $\boldsymbol{x}(E=H(\boldsymbol{x}) \geq 0)$,

2) instantaneous dissipative components for which efforts $\boldsymbol{e}_{\boldsymbol{w}}$ are related to flows $\boldsymbol{w}$ through a constitutive law $z$ that dissipates power $\left(\boldsymbol{e}_{\boldsymbol{w}}=z(\boldsymbol{w}), P_{\text {diss }}=z(\boldsymbol{w})^{\top} \boldsymbol{w} \geq\right.$ $0, \forall \boldsymbol{w})$,

3) connection ports as control inputs $\mathbf{u}$ and their associated outputs $\mathbf{y}$ such as $\mathbf{u}^{\top} \mathbf{y}$ is the outgoing power $P_{\text {ext }}$. 


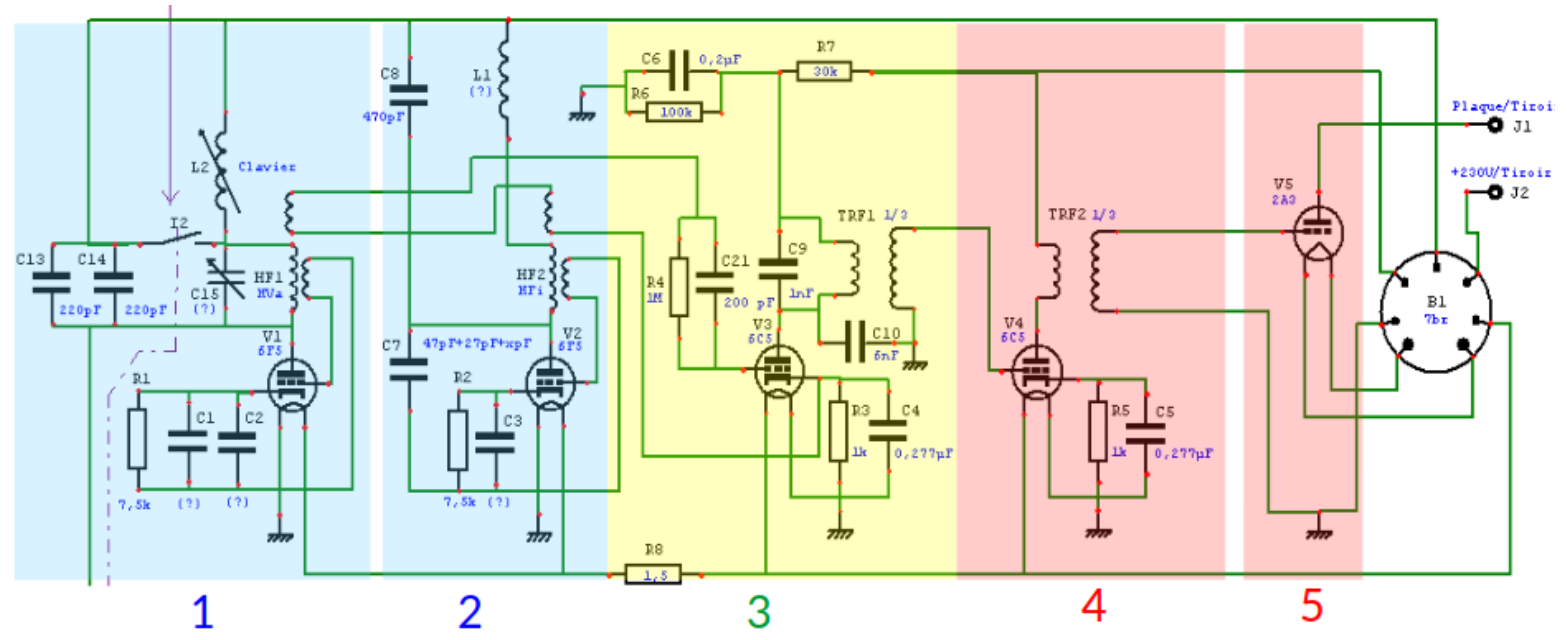

(a) Schematics of the complete circuit of the ondes No. 169 (as is from [35]).

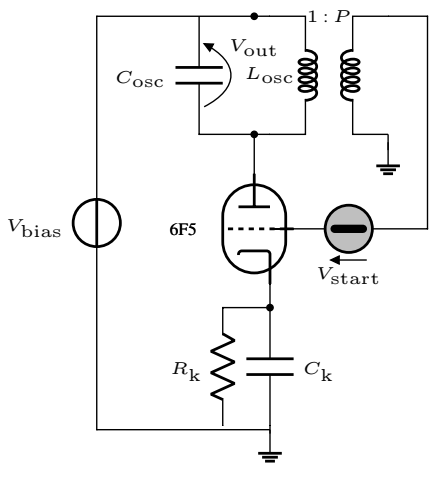

(b)

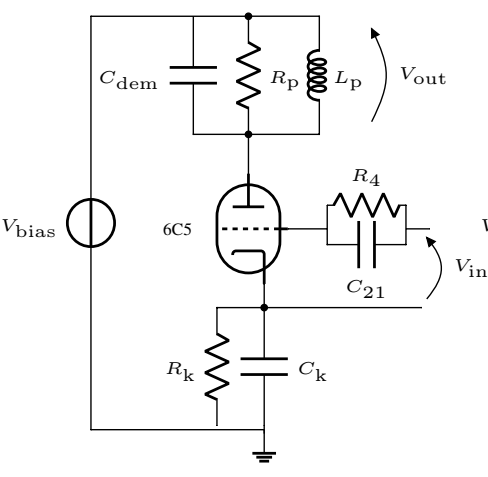

(c)

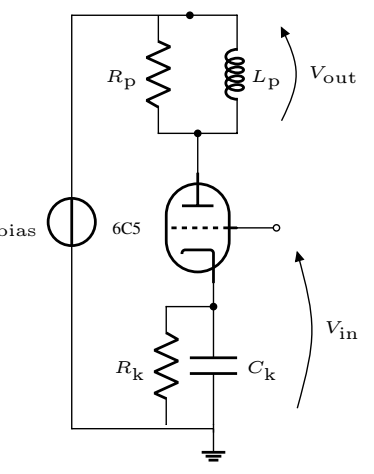

(d)

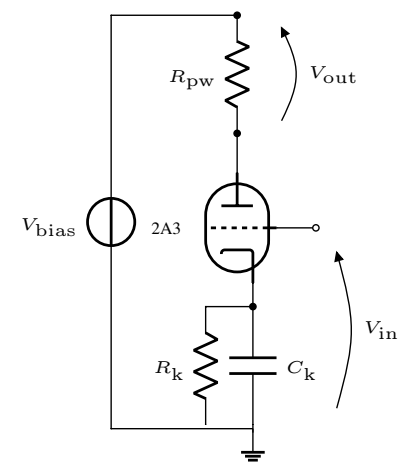

(e)

Fig. 2: Complete circuit (top) and isolated stages simplified for modeling (bottom): oscillators (stages 1 and 2, Fig. 2b), demodulator (stage 3, Fig. 2c), preamplifier (stage 4, Fig. 2d) and power amplifier (stage 5, Fig. 2e).

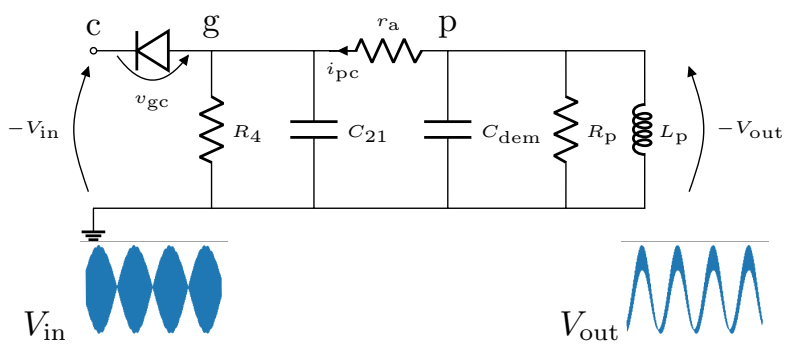

Fig. 3: Equivalent small signal schematic of the demodulator. The nodes $\mathrm{c}, \mathrm{g}$ and $\mathrm{p}$ denote the triode cathode, grid and plate respectively; $r_{\mathrm{a}}$ is the triode plate resistor.

The variables are generally time-dependent. For each component, $\dot{x}$ accounts for the incoming state flow. It is named "flow" in the following. ${ }^{1}$ Jointly, $\nabla H(x)$ is named "effort" (w.r.t. the state) in the following, so that the power received by the energy-storing component $\dot{x} \nabla H(x)$ is the product of an effort and a flow. If such a system is realizable [41], [42], the exchanges of generalized flows and efforts between the

${ }^{1}$ Note that, according to the component type, the "state flow" can be a current ( $\mathrm{i}=\dot{q}=\dot{x}$ for capacitors with charge $q$ ) or a voltage (v $=\dot{\phi}=\dot{x}$ for induction coils with magnetic flux $\phi$ ). Thus, the flow is not systematically understood as the "charge flow" that is the electric current. system components are coupled through a skew-symmetric interconnection matrix $\boldsymbol{S}=-\boldsymbol{S}^{\top}$ :

$$
\underbrace{\left(\begin{array}{c}
\dot{\boldsymbol{x}} \\
\boldsymbol{w} \\
\boldsymbol{y}
\end{array}\right)}_{\mathcal{F}(\text { flows })}=\boldsymbol{S} \underbrace{\left(\begin{array}{c}
\nabla H(\boldsymbol{x}) \\
z(\boldsymbol{w}) \\
\boldsymbol{u}
\end{array}\right)}_{\mathcal{E} \text { (efforts) }} .
$$

For convenience, to distinguish storage, dissipative, and connection ports exchanges, we introduce the following blockmatrix notation:

$$
S=\left(\begin{array}{c|c|c}
J_{\boldsymbol{x}} & -\boldsymbol{K} & -\boldsymbol{G}_{\boldsymbol{x}} \\
\hline \boldsymbol{K}^{\top} & \boldsymbol{J}_{\boldsymbol{w}} & -\boldsymbol{G}_{\boldsymbol{w}} \\
\hline \boldsymbol{G}_{\boldsymbol{x}}^{\top} & \boldsymbol{G}_{\boldsymbol{w}}^{\top} & \boldsymbol{J}_{\boldsymbol{y}}
\end{array}\right) .
$$

The skew-symmetry of $\boldsymbol{S}$ guarantees the system power balance. Indeed,

$$
\underbrace{\nabla H(\boldsymbol{x})^{\top} \dot{\boldsymbol{x}}}_{\frac{\mathrm{d} E}{\mathrm{~d} t}}+\underbrace{z(\boldsymbol{w})^{\top} \boldsymbol{w}}_{P_{\mathrm{diss}} \geq 0}+\underbrace{\boldsymbol{u}^{\top} \boldsymbol{y}}_{P_{\mathrm{ext}}}=\mathcal{E}^{\top} \mathcal{F}
$$

and

$$
\mathcal{E}^{\top} \mathcal{F}=\mathcal{E}^{\top} \boldsymbol{S} \mathcal{E}=\left(\mathcal{E}^{\top} \boldsymbol{S} \mathcal{E}\right)^{\top}=-\mathcal{E}^{\top} \boldsymbol{S} \mathcal{E}=0
$$




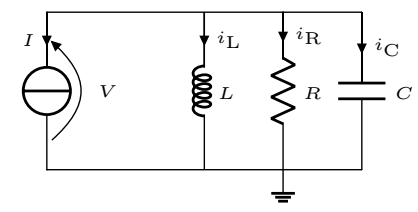

Fig. 4: Current-controlled parallel RLC circuit.

TABLE I: State variables and constitutive laws for a linear parallel RLC circuit.

\begin{tabular}{ccccc}
\hline & $x$ & $\frac{\mathrm{d} x}{\mathrm{~d} t}$ & $H(x)$ & $\nabla H(x)$ \\
\hline $\mathbf{C}$ & $q$ & $\dot{q}=i_{\mathrm{C}}$ & $\frac{q^{2}}{2 C}$ & $\frac{q}{C}=v_{\mathrm{C}}$ \\
$\mathbf{L}$ & $\phi$ & $\dot{\phi}=v_{\mathrm{L}}$ & $\frac{\phi^{2}}{2 L}$ & $\frac{\phi}{L}=i_{\mathrm{L}}$ \\
\hline & $w$ & & $z(w)$ \\
\hline $\mathbf{R}$ & & $v_{\mathrm{R}}$ & & $\frac{v_{\mathrm{R}}}{R}=i_{\mathrm{R}}$ \\
\hline
\end{tabular}

so we have the following power balance:

$$
\frac{\mathrm{d} E}{\mathrm{~d} t}=-P_{\text {ext }}-P_{\text {diss }} \text {. }
$$

Note that $-P_{\text {ext }}$ is the incoming power (since $P_{\text {ext }}$ is the outgoing power). The passivity of the system stems from this power balance and the positivity of $P_{\text {diss }}$.

\section{B. Example}

Consider a linear parallel RLC circuit (Fig. 4). The capacitor $C$ and the inductor $L$ are storage components whose states are given by the variables $q$ (charge) and $\phi$ (magnetic flux) respectively; the resistor $R$ is a dissipative component described by Ohm's law. In order to represent it as a PHS, the system is chosen to be current controlled. Table I recaps the variables and the constitutive laws of the three components. Based on Kirchoff's laws, matrix $\boldsymbol{S}$ relating efforts $(\mathcal{E})$ to flows $(\mathcal{F})$ in Eq. (1) is found to be

$$
\left(\begin{array}{c}
i_{\mathrm{C}} \\
v_{\mathrm{L}} \\
v_{\mathrm{R}} \\
V
\end{array}\right)=\left(\begin{array}{cc|c|c}
0 & -1 & -1 & -1 \\
1 & 0 & 0 & 0 \\
\hline 1 & 0 & 0 & 0 \\
\hline 1 & 0 & 0 & 0
\end{array}\right)\left(\begin{array}{c}
v_{\mathrm{C}} \\
i_{\mathrm{L}} \\
i_{\mathrm{R}} \\
I
\end{array}\right) .
$$

Flows which are currents (resp. voltages) have associated efforts which are voltages (resp. currents). Therefore their products are powers and Eq. (3) is naturally retrieved. Note that in this simple example, the matrix $\boldsymbol{S}$ is sparse with constant coefficients, but the properties of the PHS formalism also hold for non-linear or coupled systems (state depending interconnection matrices, non-quadratic $H$, non-linear $z$ ).

\section{ONDES MARTENOT PHS MOdELING}

\section{A. Ondes Martenot components}

The ondes Martenot circuit contains resistors, capacitors, inductors, transformers, and triode vacuum tubes. Among those components, the ribbon-controlled capacitor and the triode need to be carefully modeled to satisfy a physical power balance and to admit a PHS formulation.

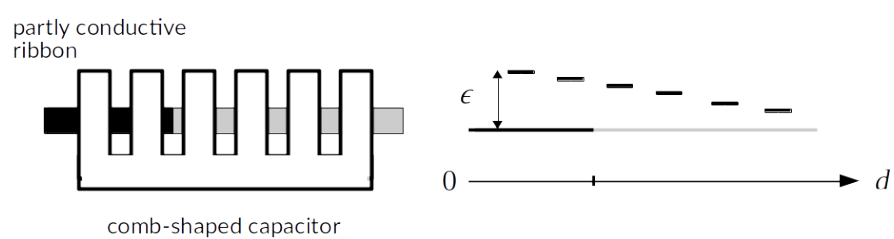

Fig. 5: Variable capacitor schematics where $d$ denotes the ribbon position (up to $1.2 \mathrm{~m}$ for the highest note) and $\epsilon(d)$ the distance between the ribbon and the comb teeth (around $1 \mathrm{~mm})$.

1) Ribbon-controlled capacitor: The variable capacitor $C_{15}$ (Fig. 2a) is controlled by a partly conductive ribbon: as the player moves the ribbon along, it hides or "activates" the teeth of the comb-shaped electrical conductor, located at different distances from the ribbon (Fig. 5). Previous work [32] showed that the LTI capacitors $C_{13}, C_{14}$ but also the variable capacitor $C_{15}$ in parallel (Fig. 2a, stage 1) are equivalent to a single variable capacitor whose energy function is given by

$$
\begin{gathered}
H_{\mathrm{cap}}(q, d)=\frac{q^{2}}{2 C_{\mathrm{osc}}(d)}, \\
C_{\mathrm{osc}}(d)=\frac{1}{4 \pi^{2}\left(F-A_{1} 2^{\frac{d}{12 d_{0}}}\right)^{2} L_{\mathrm{osc}}},
\end{gathered}
$$

where $d$ is the ribbon displacement relative to the position corresponding to the lowest note $A_{1}, d_{0}$ is the ribbon displacement for one semitone, $F$ is the fixed oscillator frequency and $L_{\text {osc }}$ is the inductance of the oscillator.

2) Triode vacuum tubes: Numerous models are available for triode vacuum tubes [43], [44]. For audio applications, the Norman Koren model [45] has proven to be accurate. Here we will rely on an enhanced Norman Koren model [46] which takes into account the triode grid current. Although this current is small compared to that of the plate, it must be considered to ensure the passivity of the component (since its power supply is external, the triode is indeed passive). As a result, the triode vacuum tube is a dissipative component with $\boldsymbol{w}=\left(v_{\mathrm{pc}}, v_{\mathrm{gc}}\right)^{\top}$ and $z_{\theta}(\boldsymbol{w})=\left(i_{\mathrm{pc}}, i_{\mathrm{gc}}\right)^{\top}$, where $p, g$ and $c$ respectively refer to the triode plate, grid and cathode. The plate current $i_{\mathrm{pc}}$ and the grid current $i_{\mathrm{gc}}$ are given by

$$
\begin{gathered}
i_{\mathrm{pc}}= \begin{cases}2\left[E_{1}\left(v_{\mathrm{pc}}, v_{\mathrm{gc}}\right)\right]^{E_{\mathrm{x}}} / K_{\mathrm{g}} & \text { if } E_{1} \geq 0 \\
0 & \text { else },\end{cases} \\
i_{\mathrm{gc}}= \begin{cases}0 & \text { if } v_{\mathrm{gc}}<V_{\mathrm{a}} \\
\frac{v_{\mathrm{gc}}-V_{\mathrm{a}}}{R_{\mathrm{gk}}} & \text { else, }\end{cases}
\end{gathered}
$$

with

$$
E_{1}\left(v_{\mathrm{pc}}, v_{\mathrm{gc}}\right)=\frac{v_{\mathrm{pc}}}{K_{\mathrm{p}}} \ln \left(1+\exp \left(K_{\mathrm{p}}\left(\frac{1}{\mu}+\frac{v_{\mathrm{gc}}+V_{\mathrm{ct}}}{\sqrt{K_{\mathrm{vb}}+v_{\mathrm{pc}}^{2}}}\right)\right)\right) .
$$

For each type of vacuum tubes used in the circuit of ondes Martenot No. 169, the set of constant parameters 


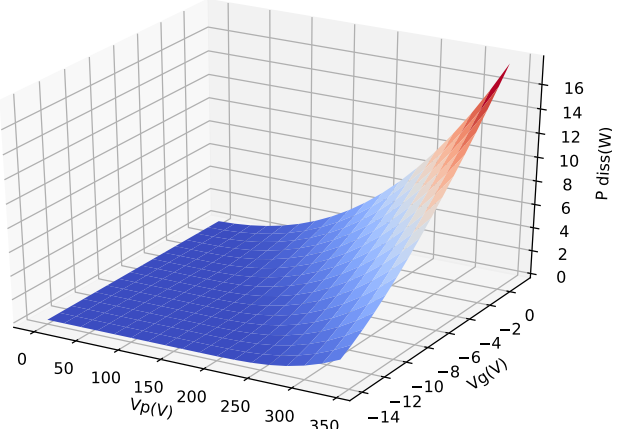

Fig. 6: Dissipated power for the triode 6C5.

$\theta=\left(\mu, E_{\mathrm{x}}, K_{\mathrm{g}}, K_{\mathrm{p}}, K_{\mathrm{vb}}, V_{\mathrm{ct}}, V_{\mathrm{a}}, R_{\mathrm{gk}}\right)$ is estimated by fitting the model to the datasheets [47, Fig. 1] [48, Fig. 1] [49, Fig. 1] through a least squares minimization. The results of that minimization fulfill the passivity criterion, i.e. $z_{\theta}(\boldsymbol{w})^{\top} \boldsymbol{w} \geq 0$ for all $\boldsymbol{w}$ (Fig. 6). They are given in Table II, section VI.

3) Other components: Constitutive laws of $R, L, C$ components are supposed to be linear, as shown in Table I. In the oscillators, the transformers are also supposed to be linear; their ratios $P$ being unknown a priori, they are subjected to an estimation described in section V-A2.

\section{B. PHS of isolated stages}

For each stage, we denote $R_{\mathrm{k}}$ the regulation resistor between the triode cathode and the ground, $C_{\mathrm{k}}$ the bypass capacitor in parallel with $R_{\mathrm{k}}, V_{\text {bias }}$ the triode DC power supply, $V_{\text {in }}$ the input voltage and $V_{\text {out }}$ the observed output voltage. We denote $L_{\mathrm{p}}$ the transformer magnetizing reactance, $R_{\mathrm{p}}$ the transformer resistance modeling the core losses in the demodulator and the preamplifier and $R_{\mathrm{pw}}$ the input impedance of the diffuseur. For each oscillator, we denote $C_{\text {osc }}$ the capacitor equivalent to the parallel capacitors and $L_{\mathrm{osc}}$ the inductance equivalent to the coils in series (Figs. 2b-2e). Kirchhoff's laws yield the interconnection matrices for the oscillator (Fig. 7a), demodulator (Fig. 7b), preamplifier (Fig. 7c), and power amplifier (Fig. 7d).

\section{Stages coupling}

The connection of two PHS is still a PHS. The total state is the concatenation of the subsystems states, and the total Hamiltonian is the sum of the subsystems Hamiltonians. A conservative interconnection is then achieved by connecting each output port of one system to exactly one input port of the other. For two stages $\mathrm{A}$ and $\mathrm{B}$ connected through a transformer of ratio $\rho$, we have $V_{\mathrm{in}_{\mathrm{B}}}=\rho V_{\mathrm{out}_{\mathrm{A}}}$ and $I_{\mathrm{out}_{\mathrm{A}}}=-\rho I_{\mathrm{in}_{\mathrm{B}}}$, that is,

$$
\left(\begin{array}{c}
y_{\mathrm{out}_{\mathrm{A}}} \\
y_{\mathrm{in}_{\mathrm{B}}}
\end{array}\right)=\left(\begin{array}{cc}
0 & 1 / \rho \\
-1 / \rho & 0
\end{array}\right)\left(\begin{array}{c}
u_{\mathrm{out}_{\mathrm{A}}} \\
u_{\mathrm{in}_{\mathrm{B}}}
\end{array}\right) .
$$

The circuit A connected to the circuit $\mathrm{B}$ is then equivalent to a circuit $\mathrm{C}$ whose PHS is given by Eq. (1), with notations of Eq. (2): $\boldsymbol{x}_{\mathrm{C}}=\left(\boldsymbol{x}_{\mathrm{A}}, \boldsymbol{x}_{\mathrm{B}}\right)^{\top}$ and $\boldsymbol{\nabla} \boldsymbol{H}_{\mathrm{C}}, \boldsymbol{w}_{\mathrm{C}}, \boldsymbol{z}_{\mathrm{C}}$ are defined likewise,

$$
J_{x_{\mathrm{C}}}=\left(\begin{array}{cc}
J_{x_{\mathrm{A}}} & 0 \\
0 & J_{x_{\mathrm{B}}}
\end{array}\right)
$$

and $\boldsymbol{J}_{\boldsymbol{w}_{\mathrm{C}}}, \boldsymbol{G}_{\boldsymbol{x}_{\mathrm{C}}}, \boldsymbol{G}_{\boldsymbol{w}_{\mathrm{C}}}, \boldsymbol{K}_{\mathrm{C}}$ are defined likewise, $\boldsymbol{u}_{\mathrm{C}}=$ $\left(\boldsymbol{u}_{\mathrm{in}_{\mathrm{A}}}, \boldsymbol{u}_{\mathrm{out}_{\mathrm{A}}}, \boldsymbol{u}_{\mathrm{in}_{\mathrm{B}}}, \boldsymbol{u}_{\mathrm{out}_{\mathrm{B}}}\right)^{\top}$ and $\boldsymbol{y}_{\mathrm{C}}$ is defined likewise, and

$$
\boldsymbol{J}_{\boldsymbol{y}_{\mathrm{C}}}=\left(\begin{array}{c|cc}
\boldsymbol{J}_{\boldsymbol{y}_{\mathrm{A}}} & 0 & 0 \\
\hline 0 & -1 / \rho & 0 \\
0 & 0 & \boldsymbol{J}_{\boldsymbol{y}_{\mathrm{B}}}
\end{array}\right) .
$$

\section{ESTIMATION OF THE INSTRUMENT PARAMETERS}

Most parameters of the components are provided by the circuit specifications [35]. The others have to be determined, in particular the oscillator transformer re-injection ratio $P$ and bypass capacitor $C_{\mathrm{k}}$, as well as the characteristics of the transformers towards the amplification stages.

\section{A. Oscillators (stages 1 and 2)}

1) Voltage noise source: The oscillation arises from the voltage noise $V_{\text {start }}$ at the grid of the triode at its operating point. This broadband noise mainly results from thermal agitation [50] and is modeled with a white noise source of peak voltage $1 \mathrm{mV}^{2}$.

2) Re-injection transformer ratio: The voltage noise is amplified by the triode, filtered by a LC circuit at the plate and sent back to the grid through a transformer of ratio $P$ (Fig. $2 b$ ). To estimate a range of possible values for $P$, the oscillator circuit is simplified: $L_{\mathrm{osc}}$ acts as the transformer primary and the cathode potential is considered constant. Moreover, the oscillator model is linearized around its operating point and it is assumed that the influence of the load on the second secondary is negligible. The oscillator can then be represented as a system of input $V_{\text {start }}$ and output $V_{\text {out }}$. Under those assumptions, it can be shown [32], [53] that the system characteristic equation in Laplace domain is $s^{2}+s(1-\mu P) /\left(r_{a} C_{\text {osc }}\right)+\omega_{0}^{2}$ ( $\mu$ being the triode amplification factor, $r_{\mathrm{a}}$ the triode plate resistor and $\omega_{0}^{2}=1 /\left(L_{\mathrm{osc}} C_{\mathrm{osc}}\right)$ the oscillation pulsation), and that the condition on $P$ for the system to oscillate is

$$
\frac{1}{\mu} \leq P<\frac{1}{\mu}+\frac{2 \omega_{0} C_{\mathrm{osc}} r_{\mathrm{a}}}{\mu}
$$

The closer to $1 / \mu P$ is chosen, the more stationary the oscillation amplitude will be. Fig. 8 shows the range of $P$ values for which an oscillation is possible.

3) Inductance of the LC circuit: For a parallel LC circuit of resonant frequency $F, L_{\mathrm{osc}}=1 /\left(C_{\mathrm{osc}}(2 \pi F)^{2}\right)$. With $F$ set to $80 \mathrm{kHz}$ and $C_{\mathrm{osc}}=544 \mathrm{pF}$, this yields $L_{\mathrm{osc}}=7 \mathrm{mH}$.

4) Cathode bypass capacitor: The bypass capacitor $C_{\mathrm{k}}$ stabilizes the cathode potential, which otherwise would depend strongly on the plate current (through the regulation resistor $R_{\mathrm{k}}$ ), itself depending on the input voltage. This stabilization ensures that the signal to be amplified $v_{\mathrm{gc}}$ is the biased input voltage and not a modulation of it. In parallel with

\footnotetext{
${ }^{2}$ The measured noise amplitude is around $1 \mu \mathrm{V}$ RMS [51] for a vacuum tube. However, as the noise is sufficiently filtered out by the oscillator, its amplitude mainly affects the transient duration [52].
} 


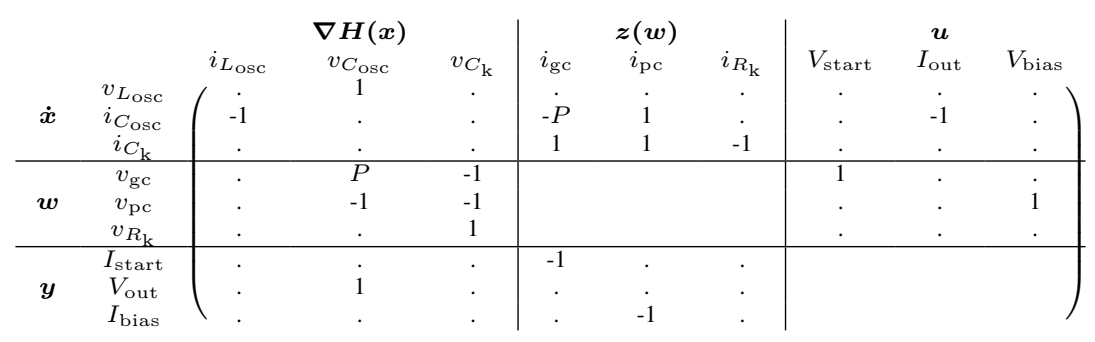

(a)

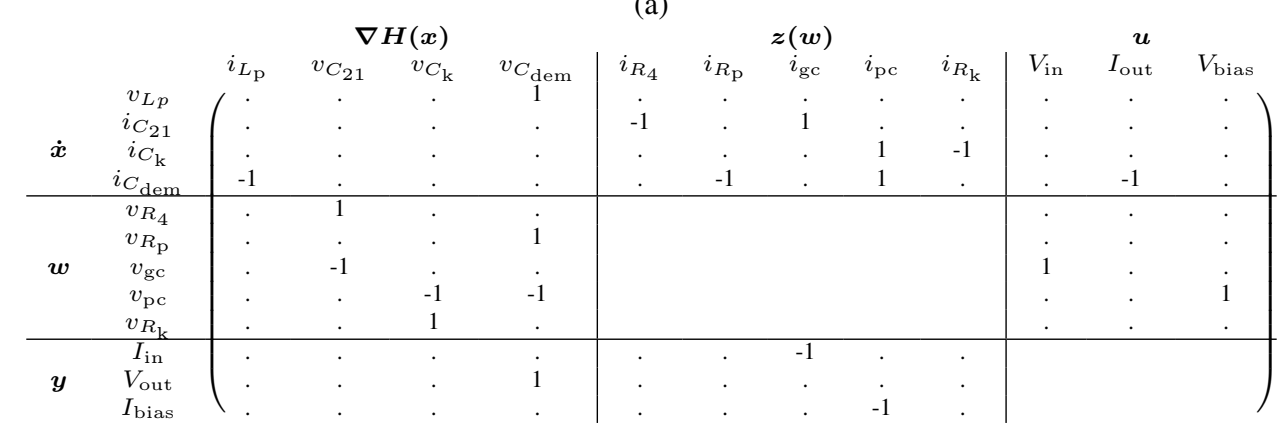

(b)

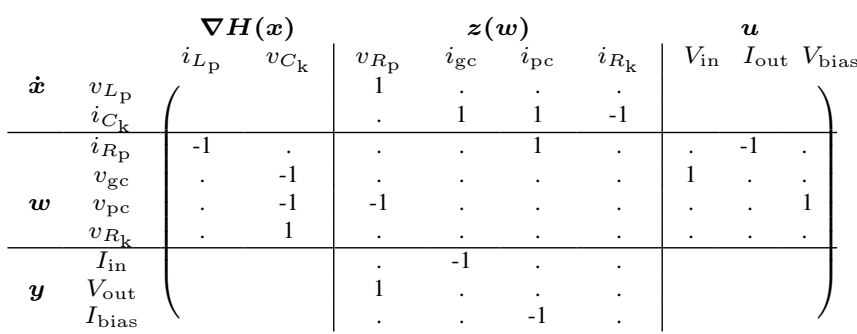

(c)

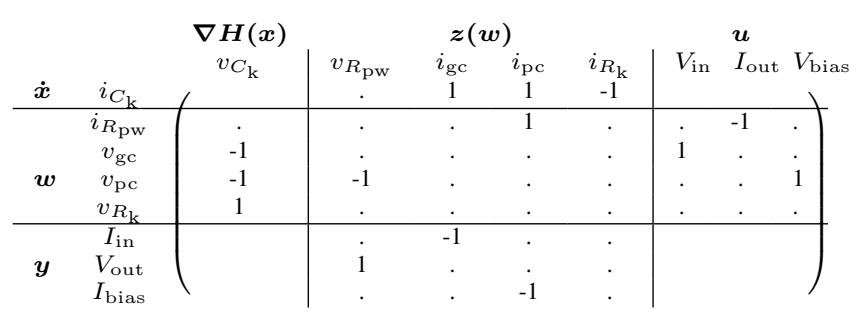

(d)

Fig. 7: PHS interconnection matrices of the isolated stages: oscillators (Fig. 7a), demodulator (Fig. 7b), preamplifier (Fig. 7c), power amplifier (Fig. 7d).

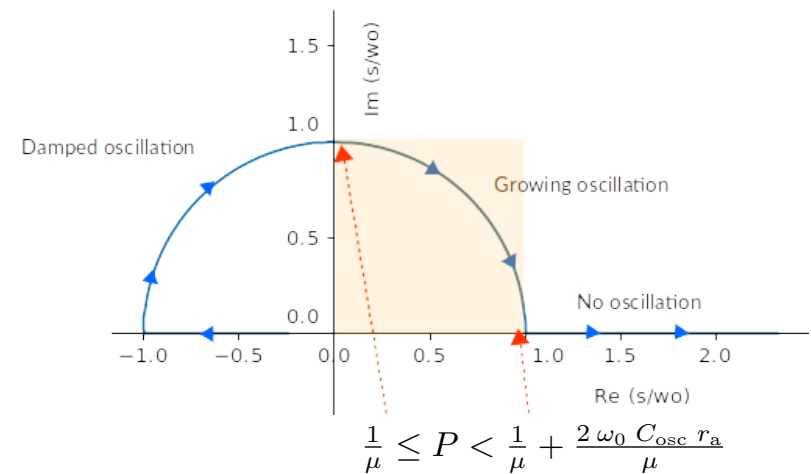

Fig. 8: Location of $s$ and regimes of oscillation for $P$ ranging between $1 / \mu$ and $1 / \mu+\beta$.

$R_{\mathrm{k}}$, it constitutes a current-current high-pass filter whose cutoff frequency is $f_{\mathrm{c}}=1 /\left(2 \pi R_{\mathrm{k}} C_{\mathrm{k}}\right)$. For the oscillator amplification to be maximal at the frequency $F, f_{\mathrm{c}}$ must verify $F \geq 10 f_{\mathrm{c}}$. For the given value $R_{\mathrm{k}}=7.5 \mathrm{k} \Omega$, this yields $C_{\mathrm{k}} \geq$ $3 \mathrm{nF}$. In the following, $C_{\mathrm{k}}$ is set to $0.22 \mu \mathrm{F}$, a typical capacitor value, for both oscillators.

\section{B. Coupling transformer characteristics}

Except for its ratio, the properties of the transformer between stage 3 (resp. 4) and 4 (resp. 5) are unknown and accessing a real circuit to perform measurements has proven to be delicate due to the instrument rarity and fragility. It is assumed that Maurice Martenot chose the transformers with audio applications in mind; the magnetizing reactance $L_{\mathrm{p}}$ and the resistance modeling the core losses $R_{\mathrm{p}}$ will be thus set as such in the following.

The capacitor $C_{\mathrm{dem}}$ in parallel with $R_{\mathrm{p}}$ and $L_{\mathrm{p}}$ (Fig. 2c) forms a current-current band-pass filter, whose high cutoff frequency is given by $f_{\mathrm{ch}}=\left(r_{\mathrm{a}}+R_{\mathrm{p}}\right) /\left(2 \pi r_{\mathrm{a}} R_{\mathrm{p}} C_{\mathrm{dem}}\right)$, with $C_{\mathrm{dem}}$ $=7 \mathrm{nF}$ and $r_{\mathrm{a}}=10 \mathrm{k} \Omega$. The instrument ambitus is C1-B7 corresponding to a fundamental frequency ranging from 32 $\mathrm{Hz}$ to $3951 \mathrm{~Hz}$ in A440 tuning. Therefore, choosing $f_{\mathrm{ch}}=$ $7.9 \mathrm{kHz}$ (the highest possible note second harmonic) yields $R_{\mathrm{p}} \approx 4 \mathrm{k} \Omega$. Similarly, the low cutoff frequency is given by $f_{\mathrm{cl}}=\left(r_{\mathrm{a}} R_{\mathrm{p}}\right) /\left(2 \pi\left(r_{\mathrm{a}}+R_{\mathrm{p}}\right) L_{\mathrm{p}}\right)$. Choosing $f_{\mathrm{cl}}=50 \mathrm{~Hz}$ yields $L_{\mathrm{p}} \approx 9 \mathrm{H}$. It is assumed that the transformer to the power amplifier has the same properties.

The values of the other circuit parameters are provided by the condition report of the ondes No. 169 [35]. 


\section{POWER-BALANCED SIMULATION}

This section is devoted to the power-balanced simulation of the ondes Martenot No. 169 complete circuit, composed of the 5 coupled stages (the power amplifier being loaded by a basic resistor equivalent to that of a diffuseur-around 1.5 $\mathrm{k} \Omega$ ). Table II recaps all components parameters values used for simulation.

TABLE II: Component parameters values used for simulation.

\begin{tabular}{|c|c|c|c|c|c|}
\hline Triode & $\mu$ & $E_{\mathrm{x}}$ & $K_{\mathrm{g}}$ & $K_{\mathrm{p}}$ & $K_{\mathrm{vb}}$ \\
\hline $6 \mathrm{F5}$ & 98 & 1.6 & 2614 & 905 & 1.87 \\
\hline $6 \mathrm{C} 5$ & 20 & 1.5 & 2837 & 138 & 89 \\
\hline \multirow[t]{2}{*}{$2 \mathrm{A3}$} & 4.3 & 1.5 & 1685 & 43 & 102 \\
\hline & $V_{\text {ct }}$ & $V_{\mathrm{a}}$ & $R_{\mathrm{gk}}$ & & \\
\hline $6 \mathrm{F5}$ & 0.5 & 0.33 & 1300 & & \\
\hline $6 \mathrm{C} 5$ & 0.8 & 0.33 & 1300 & & \\
\hline $2 \mathrm{A3}$ & -1.2 & 0.33 & 1300 & & \\
\hline Stage & $V_{\text {bias }}$ & $C_{\mathrm{k}}$ & $R_{\mathrm{k}}$ & & \\
\hline osc & $90 \mathrm{~V}$ & $0.22 \mu \mathrm{F}$ & $7.5 \mathrm{k} \Omega$ & & \\
\hline demod & $100 \mathrm{~V}$ & $0.277 \mu \mathrm{F}$ & $1 \mathrm{k} \Omega$ & & \\
\hline preamp & $180 \mathrm{~V}$ & $0.277 \mu \mathrm{F}$ & $1 \mathrm{k} \Omega$ & & \\
\hline poweramp & $230 \mathrm{~V}$ & $10 \mu \mathrm{F}$ & $750 \Omega$ & & \\
\hline$L_{\mathrm{osc}}$ & $C_{\mathrm{osc}}$ & $C_{\text {dem }}$ & $C_{21}$ & $R_{4}$ & $L_{\mathrm{p}}$ \\
\hline $7 \mathrm{mH}$ & $544 \mathrm{pF}$ & $7 \mathrm{nF}$ & $200 \mathrm{pF}$ & $1 \mathrm{M} \Omega$ & $9 \mathrm{H}$ \\
\hline$R_{\mathrm{p}}$ & $R_{\mathrm{pw}}$ & $P$ & $\rho$ & $I_{\text {out }}$ & $V_{\text {start }}$ \\
\hline $4 \mathrm{k} \Omega$ & $1.5 \mathrm{k} \Omega$ & $1 / \mu+\beta / 35$ & 3 & $0 \mathrm{~A}$ & $1 \mathrm{mV}$ \\
\hline
\end{tabular}

\section{A. Discrete-time system}

The simulation is performed using the PyPHS library [30], [54]. It is based on the numerical method recalled below in the case of mono-variate storage components $(H(\boldsymbol{x})=$ $\sum_{n=1}^{N} H_{n}\left(x_{n}\right)$ where $N$ is the storage components number). Define $\boldsymbol{x}[k]=\boldsymbol{x}\left(k / F_{s}\right)$ where $F_{s}=1 / T_{s}$ denotes the sampling rate. Moreover, define the state increment $\delta \boldsymbol{x}[k]=$ $\boldsymbol{x}[k+1]-\boldsymbol{x}[k]$ and the discrete gradient (see [30], [55]) $\bar{\nabla} H(\boldsymbol{x}[k], \delta \boldsymbol{x}[k])$, the $n^{\text {th }}$ component of which is

$$
\begin{aligned}
& {[\bar{\nabla} H(\boldsymbol{x}[k], \delta \boldsymbol{x}[k])]_{n}} \\
& = \begin{cases}\frac{H_{n}\left(x_{n}[k]+\delta x_{n}[k]\right)-H_{n}\left(x_{n}[k]\right)}{\delta x_{n}[k]} & \text { if } \delta x_{n}[k] \neq 0 \\
\frac{\mathrm{d} H_{n}}{\mathrm{~d} x_{n}}\left(x_{n}[k]\right) & \text { otherwise. }\end{cases}
\end{aligned}
$$

The method consists of replacing $\dot{\boldsymbol{x}}$ with $\delta \boldsymbol{x}[k] / T_{s}$ and $\nabla H(\boldsymbol{x})$ with $\bar{\nabla} H(\boldsymbol{x}[k], \delta \boldsymbol{x}[k])$ in Eq. (1). As in Eqs. (3-5), due to the skew-symmetry of $S$, the following discrete power balance is satisfied:

$$
\underbrace{\bar{\nabla} H(\boldsymbol{x}[k], \delta \boldsymbol{x}[k])^{\top} \frac{\delta \boldsymbol{x}[k]}{T_{s}}}_{\delta E[k] / T_{s}}+\underbrace{z(\boldsymbol{w}[k])^{\top} \boldsymbol{w}[k]}_{P_{\mathrm{diss}}[k]}+\underbrace{\boldsymbol{u}[k]^{\top} \boldsymbol{y}[k]}_{P_{\text {ext }}[k]}=0 .
$$

Moreover, due to the positivity of $z(\boldsymbol{w}[k])^{\top} \boldsymbol{w}[k]$, the system is also still passive. Denoting $\boldsymbol{\nu}=\left(\delta \boldsymbol{x}[k] F_{s}, \boldsymbol{w}[k]\right)^{\top}$, the discretization of Eq. (1) yields the implicit equation

$$
F(\boldsymbol{\nu}):=\boldsymbol{\nu}-\overline{\boldsymbol{S}} \overline{\mathcal{E}}(\boldsymbol{\nu}, \boldsymbol{x}[k], \boldsymbol{u}[k])=\mathbf{0},
$$

where

$$
\bar{S}=\left(\begin{array}{ccc}
J_{x} & -K & -G_{x} \\
K^{\top} & J_{w} & -G_{w}
\end{array}\right)
$$

and $\overline{\mathcal{E}}(\boldsymbol{\nu}, \boldsymbol{x}[k], \boldsymbol{u}[k])=(\bar{\nabla} H(\boldsymbol{x}[k], \delta \boldsymbol{x}[k]), z(\boldsymbol{w}[k]), \boldsymbol{u}[k])^{\top}$. Denoting $F^{\prime}$ the Jacobian of $F$, Eq. (12) is solved using the Newton-Raphson iteration

$$
\boldsymbol{\nu}_{k+1}=\boldsymbol{\nu}_{k}-F^{\prime}\left(\boldsymbol{\nu}_{k}\right)^{-1} F\left(\boldsymbol{\nu}_{k}\right),
$$

if $F^{\prime}$ is invertible. Conditions for convergence of the method can be found in [56], [57].

\section{B. Numerical experiments}

The circuit is simulated based on the concatenation of the stages PHS with component parameters values of Table II. The control law of the ribbon position $d$ is built to generate a frequency sweep according to parameters in Table III and Eq. (7). Fig. 9a-9c show the output voltages of stages 3, 4 and 5 during the simulation, and the effect of the successive harmonic distortions. Fig. 9d shows the spectrogram of the complete circuit output during the whole sweep. Fig. 9e shows the stored power, outgoing power and dissipated power of the complete circuit during the whole sweep. As shown in Fig. 9f, the total power, sum of these three contributions, amounts to less than $10^{-13} \mathrm{~W}$.

TABLE III: Simulation parameters.

\begin{tabular}{ll}
\hline sampling frequency $\boldsymbol{F}_{\boldsymbol{s}}$ & $768 \mathrm{kHz}$ \\
fixed osc. frequency $\boldsymbol{F}$ & $80 \mathrm{kHz}$ \\
playing frequency $\boldsymbol{f}_{\boldsymbol{m}}$ & $55 \mathrm{~Hz}-3520 \mathrm{~Hz}$ \\
duration & $1 \mathrm{~s}$ \\
\hline
\end{tabular}

\section{AUdio PLUGIN}

\section{A. Circuit simplification}

The voltage generated by the oscillators, even when connected to the other stages, is nearly sinusoidal: the harmonic distortion is about $0.03 \%$ for harmonic 2 (Fig. 10a-10b). For a real-time application, physically modeled oscillators may be thus replaced with a sinewave generator.

Additionally, although the simulated performances of the power amplifier are rather poor for a class A amplifier (almost $5 \%$ harmonic distortion for harmonic 2, Fig. 11), the stage contributions to the final sound are of much less importance compared to the demodulator and preamplifier. In practice, based on numerical experiments, this stage may also be removed for real-time application.

\section{B. Oscillators frequency reduction}

The original oscillator frequency $F$ is around $80 \mathrm{kHz}$. This choice, alongside with heterodyning, can certainly be attributed to the cost and unreliability of high value capacitors in Maurice Martenot's time: an oscillator at an audible frequency, $3000 \mathrm{~Hz}$ for instance, would necessitate a capacitor of $4 \mu \mathrm{F}$ (the inductance being equal for both oscillators). But in a virtual instrument context, the components may take 


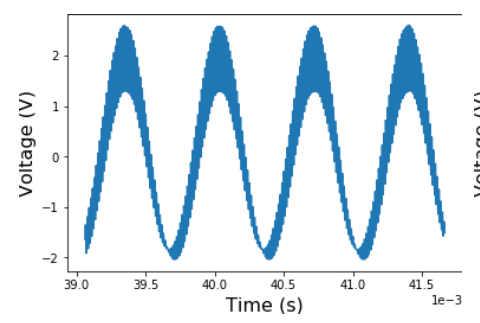

(a)

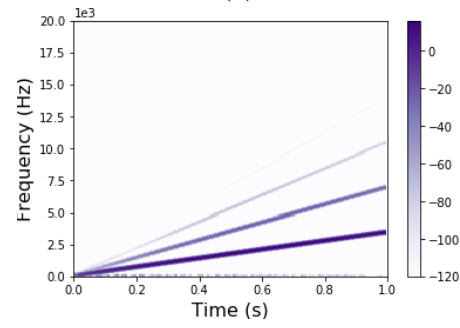

(d)

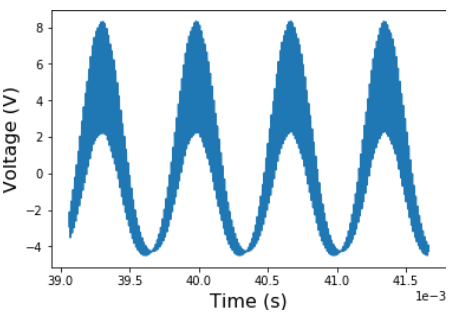

(b)

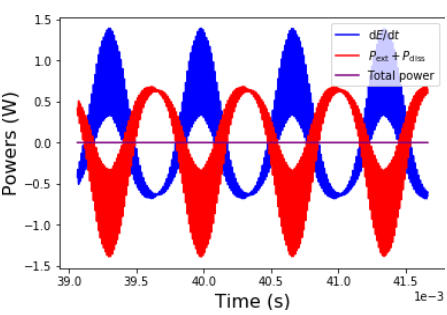

(e)

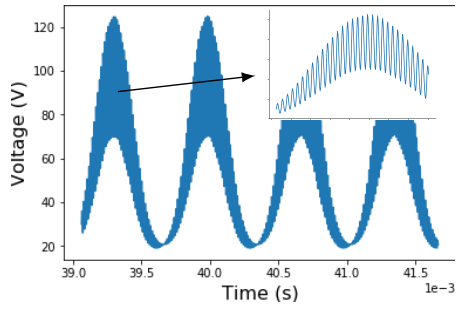

(c)

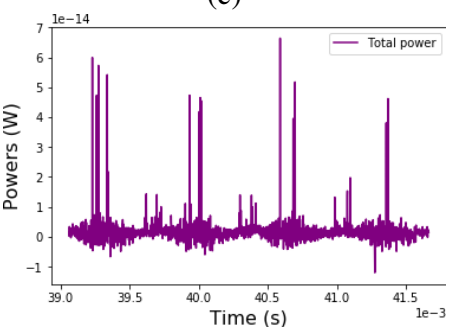

(f)

Fig. 9: From top left to bottom right: simulated output voltages at stages 3 (9a), 4 (9b) and 5 with carrier residual (9c), spectrogram of the simulated output voltage at stage 5 (9d), power balance of the circuit during the simulation (9e-9f).

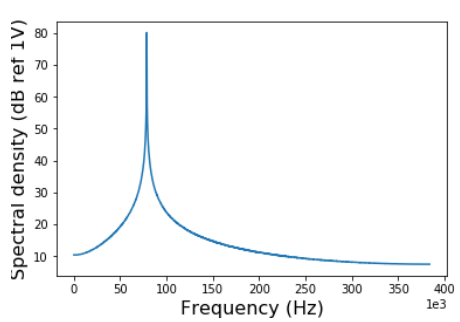

(a)

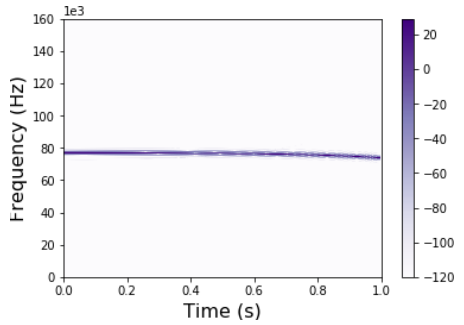

(b)

Fig. 10: Spectral density of the simulated fixed oscillator output voltage (10a) and spectrogram of the simulated variable oscillator output voltage during a sweep $\left(f_{m}=55 \mathrm{~Hz}\right.$ to $\left.3520 \mathrm{~Hz}, 10 \mathrm{~b}\right)$.

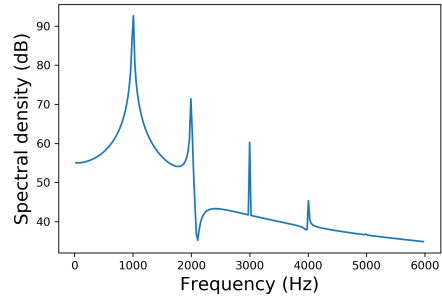

Fig. 11: Spectral density of the simulated power amplifier output voltage for an input of $1 \mathrm{kHz}$, amplitude $20 \mathrm{~V}$.

any value, provided that the oscillator frequency stays above $20 \mathrm{kHz}$. Knowing that the carrier and its first harmonics are not completely eliminated by the filtering, and that most commercial audio cards do not offer a sampling rate above $192 \mathrm{kHz}, F=48 \mathrm{kHz}$ is arbitrarily chosen to reduce the sampling rate without introducing aliasing, for real-time application. Fig. 12 shows the spectrogram of the poweramp circuit output voltage for this new configuration. A small amount of

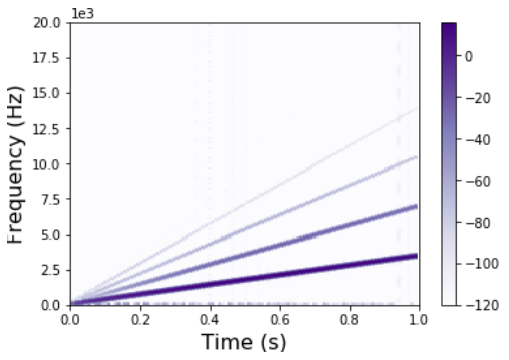

Fig. 12: Spectrogram of the simulated power amplifier output voltage for $F=48 \mathrm{kHz}$ and $F_{s}=192 \mathrm{kHz}, f_{m}=55 \mathrm{~Hz}$ to $3520 \mathrm{~Hz}$.

aliasing remains nonetheless. This can be improved either by increasing the value of $C_{\mathrm{dem}}$, therefore lowering the lowpass frequency before amplification, or implementing methods such as the ones described in [58]. Note: the detection time constant $\tau=R_{4} C_{21}=0.0002$ is still valid as the relation $\tau \gg 1 / F$ is still true. 


\section{GUI, real-time results and performances}

The dimension of the complete PHS is defined as $n=\operatorname{dim} \mathcal{E}=\operatorname{dim} \mathcal{F}$. For each sample and each element, the matrix update computes $n$ multiplications and $n-1$ additions, and consequently $F_{s} \times n(2 n-1)$ floating operations per second (flops). Before the circuit simplification, the PHS contains 32 component variables, 5 voltage supply ports, 2 inputs ports and 1 observation port. Using $F_{s}=768 \mathrm{kHz}$ and $n$ $=40$ requires 2.42 Gflops. After the circuit simplification and the frequency reduction, 17 component variables and 3 ports are removed leading to $n=20$. For $F_{s}=192 \mathrm{kHz}$, this requires 149 Mflops (gain of about 16). The simplified PHS is implemented in the JUCE framework using the PyPHS library to generate $\mathrm{C}++$ code [54]. The player controls the pitch and sound intensity either with sliders or with commercial midi interfaces. Additionally, the user has the possibility to adjust the quantity of harmonics by changing the demodulator input gain (a feature not present on the original instrument). While running, the plugin consumes $85 \% \mathrm{CPU}$ (i7-2720QM CPU at $2.20 \mathrm{GHz}$ ) and $0.9 \%$ RAM (4Go), which is still quite heavy but allows real-time usage.

\section{CONCLUSION AND PERSPECTIVES}

In this paper, a refined simulation of the ondes Martenot circuit has been proposed, allowing a numerical investigation. It is based on a power-balanced modeling adapted to LTI (capacitors, resistor, inductor) and non-LTI (vacuum tubes and the multi-physical time-varying capacitor mechanically-driven by a ribbon) components. Due to the nature of the ondes Martenot instrument (rare, fragile and expensive), setting up extensive measurements to evaluate the accuracy of the circuit modeling is a complex operation which is still ongoing. However, numerical experiments on the modeled circuit lead to the following observations:

1) the combination of the triode vacuum tube amplification properties and the LC-resonator produces a quasisinusoidal oscillation with stable amplitude;

2) the contribution of the power amplifier is minor in terms of coloration compared to that of the preamplifier;

3 ) the overall result does not depend on the carrier frequency value, provided that this frequency stays outside the audio domain.

In consequence, the oscillators are replaced with sinewave generators, the power amplifier is replaced with a multiplying factor, and the sampling frequency is lowered by a factor of 4 . These simplifications lead to the implementation of the circuit within a controllable audio plugin, functioning in real-time on a common computer.

Further work aims to model the diffuseurs and the control interfaces (ribbon and intensity key). Moreover, this work will rely on anti-aliasing methods [58] to handle the non-linear heterodyne process at lower sampling rates.

\section{ACKNOWLEDGMENTS}

This work was funded by the Collegium Musicae and is supported by the project ANR-16-CE92-0028, entitled Interconnected Infinite-Dimensional systems for Heterogeneous
Media, INFIDHEM, financed by the French National Research Agency (ANR). Further information is available at https://websites.isae-supaero.fr/infidhem/the-project. The authors thank Thierry Maniguet and Stéphane Vaiedelich (Equipe Conservation Recherche, Musée de la Musique) for their invaluable input.

\section{REFERENCES}

[1] H. Davies, "The preservation of electronic musical instruments," Journal of New Music Research, vol. 30, no. 4, pp. 295-302, 2001.

[2] A. Bonardi and J. Barthélemy, "The preservation, emulation, migration, and virtualization of live electronics for performing arts: An overview of musical and technical issues," Journal on Computing and Cultural Heritage (JOCCH), vol. 1, no. 1, p. 6, 2008.

[3] E. Leipp, "Les ondes Martenot," Bulletin du GAM No. 60, 1972.

[4] J. Pakarinen and D. T. Yeh, "A review of digital techniques for modeling vacuum-tube guitar amplifiers," Computer Music Journal, vol. 33, no. 2, pp. 85-100, 2009.

[5] G. De Sanctis and A. Sarti, "Virtual analog modeling in the wavedigital domain," IEEE Transactions on Audio, Speech, and Language Processing, vol. 18, no. 4, pp. 715-727, 2009.

[6] V. Välimäki, S. Bilbao, J. Smith, J. Abel, J. Pakarinen, and D. Berners, "Virtual analog effects," in DAFX: Digital Audio Effects. Wiley Online Library, 2011, pp. 473-522.

[7] C.-W. Ho, A. Ruehli, and P. Brennan, "The modified nodal approach to network analysis," IEEE Transactions on Circuits and Systems, vol. 22, no. 6, pp. 504-509, 1975.

[8] L. Wedepohl and L. Jackson, "Modified nodal analysis: An essential addition to electrical circuit theory and analysis," Engineering Science \& Education Journal, vol. 11, no. 3, pp. 84-92, 2002.

[9] M. Holters and U. Zölzer, "Physical modelling of a wah-wah effect pedal as a case study for application of the nodal dk method to circuits with variable parts," Proceedings of the 11th International Conference on Digital Audio Effects (DAFx-11), Paris, France, 2011.

[10] D. T. Yeh, J. S. Abel, and J. O. Smith, "Automated physical modeling of nonlinear audio circuits for real-time audio effects-part i: Theoretical development," IEEE Transactions on Audio, Speech, and Language Processing, vol. 18, no. 4, pp. 728-737, 2010.

[11] D. T. Yeh, "Automated physical modeling of nonlinear audio circuits for real-time audio effects-part ii: BJT and vacuum tube examples," IEEE Transactions on Audio, Speech, and Language Processing, vol. 20, no. 4, pp. 1207-1216, 2011.

[12] A. Fettweis, "Wave digital filters: Theory and practice," Proceedings of the IEEE, vol. 74, no. 2, pp. 270-327, 1986.

[13] S. Bilbao, Wave and scattering methods for numerical simulation. Hoboken, NJ, USA: John Wiley \& Sons, 2004.

[14] K. J. Werner, W. R. Dunkel, M. Rest, M. J. Olsen, and J. O. Smith, "Wave digital filter modeling of circuits with operational amplifiers," in 2016 24th European Signal Processing Conference (EUSIPCO), Budapest, Hungary. IEEE, 2016, pp. 1033-1037.

[15] K. J. Werner, A. Bernardini, J. O. Smith, and A. Sarti, "Modeling circuits with arbitrary topologies and active linear multiports using wave digital filters," IEEE Transactions on Circuits and Systems I: Regular Papers, vol. 65, no. 12, pp. 4233-4246, 2018.

[16] M. J. Olsen, K. J. Werner, and J. O. Smith III, "Resolving grouped nonlinearities in wave digital filters using iterative techniques," in Proceedings of the 19th International Conference on Digital Audio Effects (DAFx-16), Brno, Czech Republic, 2016.

[17] A. Bernardini, P. Maffezzoni, and A. Sarti, "Linear multistep discretization methods with variable step-size in nonlinear wave digital structures for virtual analog modeling," IEEE/ACM Transactions on Audio, Speech, and Language Processing, vol. 27, no. 11, pp. 1763-1776, 2019.

[18] A. Bernardini, A. E. Vergani, and A. Sarti, "Wave digital modeling of nonlinear 3-terminal devices for virtual analog applications," Circuits, Systems, and Signal Processing, pp. 3289-3319, 2020.

[19] Ó. Bogason and K. J. Werner, "Modeling time-varying reactances using wave digital filters," in Proceedings of the 21st International Conference on Digital Audio Effects (DAFx-18), 2018.

[20] S. D'Angelo, J. Pakarinen, and V. Valimaki, "New family of wave-digital triode models," IEEE Transactions on Audio, Speech, and Language Processing, vol. 21, no. 2, pp. 313-321, 2012. 
[21] J. Pakarinen, M. Tikander, and M. Karjalainen, "Wave digital modeling of the output chain of a vacuum-tube amplifier," in Proceedings of the 12th International Conference on Digital Audio Effects (DAFx-09), Como, Italy, 2009.

[22] K. Dempwolf, M. Holters, and U. Zölzer, "Discretization of parametric analog circuits for real-time simulations," in Proceedings of the 13th International Conference on Digital Audio Effects (DAFx-10), Graz, Austria, 2010.

[23] F. Fontana and M. Civolani, "Modeling of the EMS VCS3 voltagecontrolled filter as a nonlinear filter network," IEEE Transactions on Audio, Speech, and Language Processing, vol. 18, no. 4, pp. 760-772, 2010.

[24] I. Cohen and T. Hélie, "Real-time simulation of a guitar power amplifier," in Proceedings of the 13th International Conference on Digital Audio Effects (DAFx-10), Graz, Austria, 2010.

[25] J. Macak, J. Schimmel, and M. Holters, "Simulation of Fender type guitar preamp using approximation and state space model," in Proceedings of the 10th International Conference on Digital Audio Effects (DAFx12), York, UK, 2012.

[26] B. M. Maschke, A. J. Van der Schaft, and P. Breedveld, "An intrinsic Hamiltonian formulation of network dynamics: Non-standard Poisson structures and gyrators," Journal of the Franklin Institute, pp. 923-966, 1992.

[27] A. Van der Schaft, D. Jeltsema, et al., "Port-Hamiltonian systems theory:

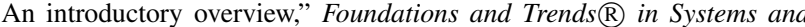
Control, vol. 1, no. 2-3, pp. 173-378, 2014.

[28] N. Lopes, T. Hélie, and A. Falaize, "Explicit second-order accurate method for the passive guaranteed simulation of port-Hamiltonian systems," IFAC-PapersOnLine, vol. 48, no. 13, pp. 223-228, 2015.

[29] N. Lopes and T. Hélie, "Energy balanced model of a jet interacting with a brass player's lip," Acta Acustica united with Acustica, vol. 102, no. 1, pp. 141-154, 2016

[30] A. Falaize and T. Hélie, "Passive guaranteed simulation of analog audio circuits: A port-Hamiltonian approach," Applied Sciences, vol. 6, no. 10, p. 273, 2016.

[31] _ "Passive simulation of the nonlinear port-Hamiltonian modeling of a Rhodes piano," Journal of Sound and Vibration, vol. 390, pp. 289309, 2017.

[32] J. Najnudel, T. Hélie, and D. Roze, "Simulation of the ondes Martenot ribbon-controlled oscillator using energy-balanced modeling of nonlinear time-varying electronic components," Journal of the Audio Engineering Society, vol. 67, no. 12, pp. 961-971, 2019.

[33] J. Laurendeau, Maurice Martenot, luthier de l'électronique. SaintZenon, Quebec, Canada: Louise Courteau, 1990

[34] S. Ramel, "Conservation and restoration of electroacoustic musical instruments at the Musée de la Musique, Paris," Organised Sound, vol. 9, no. 1, pp. 87-90, 2004.

[35] T. Courrier, "Analyse de fonctionnement onde 169," 2012, unpublished document, Musée de la Musique.

[36] A. Glinsky and B. Moog, Theremin: Ether music and espionage. Champaign, IL, USA: University of Illinois Press, 2000.

[37] P. Couprie, "Oskar sala," Observatoire Leonardo des Arts et Technosciences, 2002.

[38] L. Quartier, T. Meurisse, J. Colmars, J. Frelat, and S. Vaiedelich, "Intensity Key of the Ondes Martenot: An Early Mechanical Haptic Device," Acta Acustica united with Acustica, vol. 101, no. 2, pp. 421-428, 2015. [Online]. Available: https://hal.sorbonne-universite.fr/ hal-01587642

[39] J. S. Belrose, "Reginald Aubrey Fessenden and the birth of wireless telephony," IEEE Antennas and Propagation Magazine, vol. 44, no. 2, pp. 38-47, 2002.

[40] I. Cohen, "Modélisation, analyse et identification de circuits non linéaires: Application aux amplificateurs guitare à lampes pour la simulation en temps réel,” Ph.D. dissertation, Université Pierre \& Marie Curie-Paris 6, 2012.

[41] R. W. Brockett, Finite dimensional linear systems. Philadelphia, PA, USA: SIAM, 2015, vol. 74 .

[42] A. Van der Schaft, "A realization procedure for systems of nonlinear higher-order differential equations," IFAC Proceedings Volumes, vol. 20, no. 5 , pp. 85-90, 1987.

[43] W. M. Leach Jr, "Spice models for vacuum-tube amplifiers," Journal of the Audio Engineering Society, vol. 43, no. 3, pp. 117-126, 1995.

[44] G.-C. Cardarilli, M. Re, and L. Di Carlo, "Improved large-signal model for vacuum triodes," in 2009 IEEE International Symposium on Circuits and Systems. IEEE, 2009, pp. 3006-3009.

[45] N. Koren, "Improved vacuum tube models for Spice simulations," Glass Audio, vol. 8, no. 5, pp. 18-27, 1996.
[46] I. Cohen and T. Hélie, "Measures and parameter estimation of triodes, for the real-time simulation of a multi-stage guitar preamplifier," in 129th Convention of Audio Engineering Society, San Francisco, United States, Nov. 2010. [Online]. Available: https://hal.archives-ouvertes.fr/hal-00631747

[47] Tung-Sol, "6F5 typical operating conditions and characteristics," https: //frank.pocnet.net/sheets/127/6/6F5.pdf, 1942.

[48] — " "6C5 typical operating conditions and characteristics," https: //frank.pocnet.net/sheets/127/6/6C5.pdf, 1939.

[49] RCA, "2A3 average plate characteristics," https://frank.pocnet.net/ sheets/049/2/2A3.pdf, 1933.

[50] F. Llewellyn, "A study of noise in vacuum tubes and attached circuits," Proceedings of the Institute of Radio Engineers, vol. 18, no. 2, pp. 243 $265,1930$.

[51] G. Pearson, "Fluctuation noise in vacuum tubes," Bell System Technical Journal, vol. 13, no. 4, pp. 634-653, 1934.

[52] W. A. Edson, "Noise in oscillators," Proceedings of the IRE, vol. 48, no. 8, pp. 1454-1466, 1960 .

[53] Y. Rocard, Dynamique générale des vibrations. Massoncie, 1971.

[54] A. Falaize and T. Hélie, "PyPHS: Passive modeling and simulation in python," 2016, python package, Web page. [Online]. Available: https://hal.archives-ouvertes.fr/hal-01470383

[55] T. Itoh and K. Abe, "Hamiltonian-conserving discrete canonical equations based on variational difference quotients," Journal of Computational Physics, vol. 76, no. 1, pp. 85-102, 1988.

[56] R. Muller and T. Hélie, "Power-Balanced Modelling Of Circuits As Skew Gradient Systems," in 21 st International Conference on Digital Audio Effects (DAFx-18), Aveiro, Portugal, Sept. 2018. [Online]. Available: https://hal.archives-ouvertes.fr/hal-01871464

[57] P. Deuflhard, Newton methods for nonlinear problems: affine invariance and adaptive algorithms. Springer Science \& Business Media, 2011, vol. 35.

[58] R. Muller and T. Hélie, "Trajectory anti-aliasing on guaranteed-passive simulation of nonlinear physical systems," in Proceedings of the 20th International Conference on Digital Audio Effects (DAFx-17), Edinburgh, $U K, 2017$.

Judy Najnudel is graduated from Ecole Nationale Supérieure Louis Lumière (2007) and Sorbonne University (ATIAM MSc in 2018). She is currently pursuing a $\mathrm{PhD}$ at the STMS laboratory (Sciences and Technologies for Music and Sound, IRCAM-CNRS-SU). She focuses on physical modeling of nonlinear analog circuits for audio applications.

Thomas Hélie is graduated from the Ecole Nationale Supérieure des Télécommunications de Bretagne (Ing. Dipl. in 1997), University Paris VI (ATIAM MSc in 1998 and Habilitation à diriger des Recherches in 2013) and University Paris XI (MSc ATS in 1998 and PhD in 2002). Since 2017, he is director of research at CNRS, head of the S3AM team at STMS laboratory and coordinator of the ATIAM MSc. His research topics include nonlinear dynamical system and control theory, signal processing, acoustics, and physical modeling of audio and musical instruments.

David Roze is graduated from INSA de Rouen (Ing. Dipl. in 2005) and University Paris VI (ATIAM MSc in 2006 and PhD in 2010). He is a CNRS researcher at STMS laboratory. His research interests include control of nonlinear dynamical systems, sound synthesis, and numerical methods for real-time computation.

Henri Boutin is graduated in electrical engineering and acoustics from ENSEA, Cergy, France, Universidad Politécnica de Madrid (Eng. Dipl. in 2005 and 2008), and from University Paris VI (ATIAM MSc 2006 and PhD in 2011). As a lecturer at Sorbonne University, Paris, he focuses his research on the acoustics, vibration and control of musical instruments and cultural heritage objects. 